\title{
Influence of Multiscale Roughness Patterns in Cavitated Flows: Applications to Journal Bearings
}

\author{
Sébastien Martin \\ Laboratoire de Mathématiques, Université Paris-Sud XI, CNRS-UMR 8628, Bâtiment 425, \\ 91405 Orsay Cedex, France \\ Correspondence should be addressed to Sébastien Martin, sebastien.martin@math.u-psud.fr
}

Received 2 November 2007; Accepted 19 March 2008

Recommended by Giuseppe Rega

This paper deals with the coupling of two major problems in lubrication theory: cavitation phenomena and roughness of the surfaces in relative motion. Cavitation is defined as the rupture of the continuous film due to the formation of air bubbles, leading to the presence of a liquid-gas mixture. For this, the Elrod-Adams model (which is a pressure-saturation model) is classically used to describe the behavior of a cavitated thin film flow. In addition, in practical situations, the surfaces of the devices are rough, due to manufacturing processes which induce defaults. Thus, we study the behavior of the solution, when highly oscillating roughness effects on the rigid surfaces occur. In particular, we deal with the reiterated homogenization of this Elrod-Adams problem, using periodic unfolding methods. A numerical simulation illustrates the behavior of the solution. Although the pressure tends to a smooth one, the saturation oscillations are not damped. This does not prevent us from defining an equivalent homogenized saturation and highlights the anisotropic effects on the saturation function in cavitated areas.

Copyright (C) 2008 Sébastien Martin. This is an open access article distributed under the Creative Commons Attribution License, which permits unrestricted use, distribution, and reproduction in any medium, provided the original work is properly cited.

\section{Introduction}

A journal bearing, simply stated, is made of an external cylinder which surrounds a rotating shaft and is filled with some form of fluid lubricant that supports the shaft preventing metal to metal contact. In this framework, the Reynolds equation has been used for a long time to describe the behavior of this type of flows (see Reynolds [1] for historical references) using the pressure $P$ in the thin film as the leading unknown in the problem.

However, the Reynolds modelling does not take into account cavitation phenomena: cavitation is defined as the rupture of the continuous film due to the formation of gas bubbles and makes the Reynolds equation no longer valid in the cavitation area. In order to make it possible, we use the Elrod-Adams model, which introduces the hypothesis that the cavitation region is a liquid-gas mixture and an additional unknown $\theta$ (the saturation of liquid in the 
mixture) (see Capriz and Cimatti [2], Coyne and Elrod [3, 4], and Elrod and Adams [5]). The model, which still relies on the Reynolds equation, is widely used in tribology and appears to give satisfactory results with respect to mechanical experiments. The interest of this model also relies on the fact that it is a mass-preserving model, unlike some others such as the variational inequalities model.

Finally, the effects of the surface roughness on the behavior of a thin film flow have gained an increasing attention from 1960 since it was thought to be an explanation for the unexpected load support in bearings. The roughness defaults can be modelled with a parameter $\varepsilon$, which denotes the typical spacing between two patterns; as the number of patterns increases, numerical costs tend to explode because of mesh refinements required for the description of the gap. The effect of periodic roughness has been treated by means of a homogenization procedure in numerous works depending on the lubrication regimes: let us mention the works of Patir and Cheng [6] for the linear case (without cavitation), Jai [7] for compressible thin films flows, and Bayada and Faure [8] for a cavitated flow using a variational inequalities model. Some of these theoretical studies include numerical examples which show how significant pressure perturbations appear, due to the presence of surface asperities. So far, in all these works, the roughness patterns were modelled by only one typical pattern, corresponding to one type of defaults. This assumption is reasonable for many mechanical applications, but it lacks relevance as manufacturing processes may lead to different defaults at different lengthscales (namely, $\varepsilon$ and $\varepsilon^{2}, \ldots$ ) by using a polishing solution containing metal oxide abrasive grains to form a surface. Moreover, multiscale roughness patterns may be introduced in a voluntary way, the motivation for this being related to shape optimization of the surfaces, load experiments, and control of the friction. Thus, it is the purpose of this paper to focus on the influence of the roughness effects in the framework of reiterated homogenization dealing with a more realistic model of cavitated thin films flow.

The paper is organized as follows. Section 2 is devoted to the description of thin film flows in journal bearings. Section 3 deals with the reiterated homogenization process of the problem. Section 4 presents a numerical simulation which illustrates the main results of the previous section. Additionally, Appendix A provides the main tools and results related to the periodic unfolding method which is used in this paper and Appendix B presents the detailed procedure for the computation of the homogenized coefficients in a particular but realistic case.

\section{Thin film flows in journal bearings}

\subsection{Hydrodynamic lubrication}

Journal bearings are among the most common lubricated devices. A journal bearing (see Figure 1) is made of an external cylinder which surrounds a rotating shaft (or internal cylinder, or journal) and is filled with some form of fluid lubricant. The most common fluid used is oil, with special applications using water or a gas. Geometrical data are the following ones: $L$ is the length of the cylinders, $R_{b}$ (resp., $R_{j}$ ) is the section radius for the external cylinder (resp., shaft), and $R_{m}=\left(R_{b}+R_{j}\right) / 2$ is the average radius. Let us introduce $c=R_{b}-R_{j}$ the radial clearance, $e$ the eccentricity, and $\omega$ the angular speed of the rotating shaft. The two cylinders are closely spaced and the smallness of this ratio allows for a Cartesian coordinate to be located on the bearing surface. In this fictitious setting (corresponding to an artificial cut located at the supply groove, which leads to a developed configuration of the device, see Figure 1 on the one 


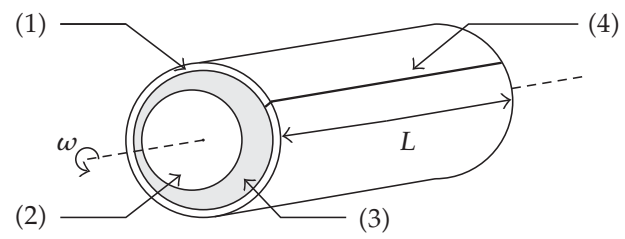

Figure 1: Journal bearing device: (1) external cylinder, with radius $R_{b}$, (2) shaft, with radius $R_{j}$, (3) lubricant, and (4) axial supply groove $\Gamma_{\star}$.

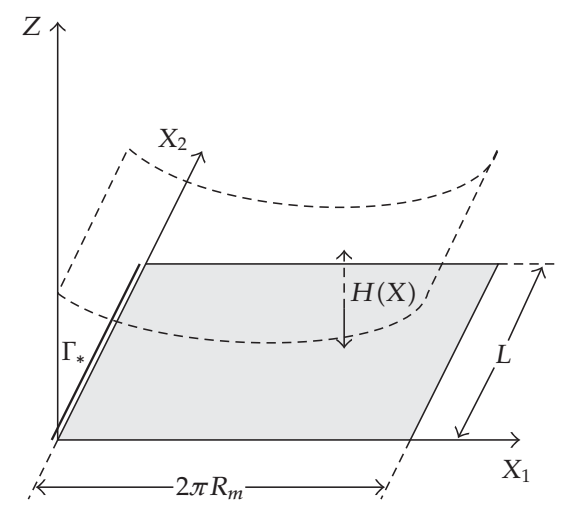

Figure 2: Fictitious developed domain in real coordinates.

hand and Figure 2 on the other hand), the gap between the two surfaces is given by

$$
H(X)=c+e \cos \left(\frac{X_{1}}{R_{m}}\right) \text {. }
$$

The Reynolds equation has been used for a long time to describe the behavior of a viscous flow between two close surfaces in relative motion (see the work of Reynolds [1] for historical references). The transition of the Stokes equation to the Reynolds equation has been proved in a rigorous way by Bayada and Chambat [9]. In real variables, the classical Reynolds problem should be posed as

$$
\left.\operatorname{div}\left(\frac{H^{3}}{6 \mu} \nabla P\right)=v_{0} \frac{\partial}{\partial X_{1}}(H), \quad \text { in }\right] 0,2 \pi R_{m}[\times] 0, L[,
$$

where $P$ is the (unknown) pressure distribution, $\mu$ the lubricant viscosity, $v_{0}=\omega R_{m}$ the shearing velocity, and $H$ the gap between the surfaces. Now let us introduce the dimensionless coordinates and quantities that provide the reduced system to solve

$$
x_{1}=\frac{X_{1}}{2 \pi R_{m}}, \quad x_{2}=\frac{X_{2}}{L}, \quad h(x)=\frac{H(X)}{c}, \quad p=\frac{c^{2} P}{6 \mu v_{0} 2 \pi R_{m}}, \quad \kappa=\frac{2 \pi R_{m}}{L} .
$$

Then, the dimensionless Reynolds equation becomes

$$
\left.\frac{\partial}{\partial x_{1}}\left(h^{3} \frac{\partial p}{\partial x_{1}}\right)+\kappa^{2} \frac{\partial}{\partial x_{2}}\left(h^{3} \frac{\partial p}{\partial x_{2}}\right)=\frac{\partial}{\partial x_{1}}(h), \quad \text { in } \Omega=\right] 0,1[\times] 0,1[
$$

where $p$ is the normalized pressure distribution, and $h$ the normalized gap between the two 
surfaces ( $h$ is assumed to be a regular positive function). Without loss of generality, we will assume that $\kappa=1$ (this does not alter the mathematical structure of the problem).

\subsection{Cavitation}

Let us introduce cavitation phenomena: in diverging profiles of the flow, the pressure may decrease until it reaches the vapor pressure, thus leading to the formation of gas bubbles at a near constant pressure (the vapor pressure). In order to take it into account, the Elrod-Adams model modifies the Reynolds equation by introducing an additional leading unknown $\theta$ (the saturation of liquid in the mixture) (see [2-5]):

$$
\begin{aligned}
& \operatorname{div}\left(h^{3} \nabla p\right)=\frac{\partial}{\partial x_{1}}(\theta h), \\
& p \geq p_{s}, \theta \in H\left(p-p_{s}\right),
\end{aligned}
$$

where $H$ denotes the Heaviside graph. Here, the vapor pressure $p_{s}$ will be taken equal to the ambient pressure $p_{a}=0$ :this is justified by the fact that hydrodynamic pressure $p$ is high so that $p_{s}-p_{a}$ can be neglected with respect to $p-p_{a}$. Notice that the model introduces a free boundary which separates two different areas:

(a) in the saturated regions, $p>p_{s}, \theta=1$, (classical Reynolds equation);

(b) in cavitated regions, $p=p_{s}, 0 \leq \theta \leq 1$, (partial lubrication).

Thus, $\theta$ describes the local ratio of the liquid phase between the two surfaces.

\subsection{Boundary conditions}

We consider a rectangular domain $\Omega=] 0,1[\times] 0,1\left[; \Gamma_{\star}\right.$ denotes the boundary $\left.\{0\} \times\right] 0,1[$ and $\Gamma=\partial \Omega \backslash \Gamma_{\star}$ (see Figure 2, up to the normalization procedure). The boundary conditions are strongly related to the following remarks:

(1) the boundary $\Gamma_{\star}$ corresponds to the (left part of the) supply groove; thus, an input flow (Neumann conditions) is considered, the flow being advected by the shear (from the left to the right);

(2) the boundary $\{1\} \times(0,1)$ corresponds to the (right part of the) supply groove: this open boundary is thus associated to Dirichlet conditions (ambient pressure);

(3) the boundaries $(0,1) \times\{0\}$ and $(0,1) \times\{1\}$ are the upper and lower bases of the surrounding cylinders: open walls impose Dirichlet conditions (ambient pressure).

This configuration is related to the following set of boundary conditions:

$$
\begin{gathered}
p=0 \quad \text { on } \Gamma, \\
\theta h-h^{3} \frac{\partial p}{\partial x_{1}}=Q \quad \text { on } \Gamma_{\star} .
\end{gathered}
$$

Here, $Q$ denotes the input flow, which may be classically normalized as

$$
Q=\theta_{\star} \int_{0}^{1} h(0, \cdot), \quad \text { with } \theta_{\star} \in[0,1] .
$$




\subsection{Variational formulation of (2.5)-(2.7)}

The initial problem for (2.5)-(2.7) should be mathematically analyzed with the following variational formulation:

$$
\left(D_{\theta}\right)\left\{\begin{array}{l}
\text { find }(p, \theta) \in V \times L^{\infty}(\Omega) \text { such that } \\
\int_{\Omega} h^{3} \nabla p \nabla \phi=\int_{\Omega} \theta h \frac{\partial \phi}{\partial x_{1}}+\int_{\Gamma_{\star}} Q \phi, \quad \forall \phi \in V, \\
p \geq 0, \quad \theta \in H(p), \quad \text { a.e., }
\end{array}\right.
$$

where the functional space $V$ is defined by

$$
V=\left\{v \in H^{1}(\Omega), v_{\mid \Gamma}=0\right\}
$$

Problem $\left(D_{\theta}\right)$ is well-posed: it admits a unique solution (see [10-12] for details) and algorithms are known to solve the problem (see, e.g., the papers by Alt [13], Bayada et al. [14], Marini and Pietra [15]).

\subsection{Influence of roughness defaults}

The effects of the surface roughness on the behavior of a thin film flow have long been the subject of intensive studies. The roughness defaults (whose typical amplitude is given) can be modelled with the introduction of a small parameter $\varepsilon$, which denotes the typical spacing between two patterns. In this framework, gap functions become highly oscillating. Of course, the introduction of small parameters, for the description of the roughness patterns, leads to heavy computational costs which can be avoided by considering the asymptotic problem as the so-called homogenization process aims at avoiding those difficulties by considering an equivalent averaged problem (with smoother coefficients) whose solution can be computed more easily. In this way, the effect of periodic roughness on the behavior of hydrodynamic magnitudes has been treated in numerous works depending on the lubrication regimes (see, e.g., [6-8]). However, in all these works, the roughness patterns were modelled by only one typical (periodic) pattern, corresponding to one type of defaults. This assumption is not necessarily reasonable for many mechanical applications as manufacturing processes may lead to different defaults, characterized by different lengthscales. Typically, we take into account patterns modelled by two different scales (namely, $\varepsilon$ and $\varepsilon^{2}$ ) so that the gap function takes the form (see Figure 3)

$$
h:=h\left(x, \frac{x}{\varepsilon}, \frac{x}{\varepsilon^{2}}\right),
$$

leading to coupling effects between the all micro- and macroscales $\varepsilon^{2}, \varepsilon$, and 1 . Here, we restrict ourselves to this type of multiscale defaults in order to study the mathematical structure of the limit problem. However, the generalization to more complicated patterns, involving surface defaults at scales $\varepsilon, \varepsilon^{2}, \varepsilon^{3}$, and so forth, will be straightforward. 


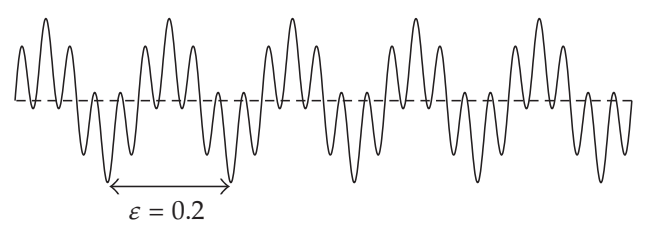

(a)

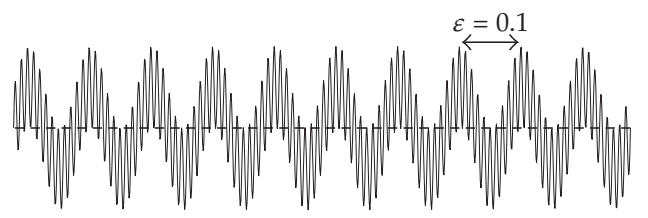

(b)

Figure 3: Roughness patterns at scales $\varepsilon$ and $\varepsilon^{2}$ on a planar one-dimensional surface.

\section{Reiterated homogenization of the problem}

We now make precise the roughness patterns considered. The effective gap is now described by a nominal regular thickness to which one adds the roughness defaults around the averaged gap. We thus consider a gap of the form

$$
h_{\varepsilon}(x)=h\left(x, \frac{x}{\varepsilon}, \frac{x}{\varepsilon^{2}}\right)
$$

where $h \in L^{\infty}\left(\Omega, C_{\#}^{1}\left(\left[0,1[)^{4}\right)\right.\right.$ satisfies the additional assumption

$$
\exists \underline{h}, \bar{h}, \quad 0<\underline{h} \leq h_{\varepsilon} \leq \bar{h} .
$$

This assumption leads to consider two roughness scales $\varepsilon$ and $\varepsilon^{2}$. The definition of the gap given by (3.1) leads to the interaction between the scales $\varepsilon$ and $\varepsilon^{2}$.

Our goal is to describe the asymptotic behavior of the solution $\left(p_{\varepsilon}, \theta_{\varepsilon}\right)$ of problem $\left(\mathcal{D}_{\theta}^{\varepsilon}\right)$. For this, we study the convergence of the solution and determine the homogenized equations satisfied by the limit functions, by means of the periodic unfolding method (see Appendix A).

\subsection{Micro-/macrodecomposition}

Proposition 3.1. There exist $\left(p_{0}, p_{1}, p_{2}\right) \in V \times L^{2}\left(\Omega ; H_{\#}^{1}(Y) / \mathbb{R}\right) \times L^{2}\left(\Omega \times Y ; H_{\#}^{1}(Z) / \mathbb{R}\right)$ and $\theta_{0} \in$ $L^{2}(\Omega \times Y \times Z)$ such that, up to a subsequence, the following convergences hold in $L^{2}(\Omega \times Y \times Z)$ :

$$
\begin{gathered}
\tau_{\varepsilon \circ \varepsilon}\left(p_{\varepsilon}\right) \longrightarrow p_{0}, \\
\tau_{\varepsilon \circ \varepsilon}\left(\nabla p_{\varepsilon}\right)-\nabla p_{0}+\nabla_{y} p_{1}+\nabla_{z} p_{2}, \\
\tau_{\varepsilon \circ \varepsilon}\left(\theta_{\varepsilon}\right)-\theta_{0} .
\end{gathered}
$$

Proof. It can be easily proved that $p_{\varepsilon}$ (resp., $\theta_{\varepsilon}$ ) is bounded in $H^{1}(\Omega)$ (resp., $L^{2}(\Omega)$ ). Then, by Proposition A.3, the convergence results are straightforward. 
Proposition 3.2. $p_{0} \geq 0$ and $\theta_{0} \in H\left(p_{0}\right)$ a.e.

Proof. We proceed in two steps as follows.

Step 1. As $p_{\varepsilon} \geq 0,0 \leq \theta_{\varepsilon} \leq 1$ a.e. and using the definition of the unfolding operator, one has $\tau_{\varepsilon}\left(p_{\varepsilon}\right) \geq 0$ and $0 \leq \tau_{\varepsilon}\left(\theta_{\varepsilon}\right) \leq 1$ a.e. The convergences' properties of Proposition 3.1 then imply

$$
p_{0}(x) \geq 0, \quad 0 \leq \theta_{0}(x, y, z) \leq 1, \quad \text { for a.e. }(x, y, z) \in \Omega \times Y \times Z .
$$

Step 2. Applying the unfolding operator to each side of the equality $p_{\varepsilon}\left(1-\theta_{\varepsilon}\right)=0$ and passing to the limit, we get $p_{0}\left(1-\theta_{0}\right)=0$ in $L^{1}(\Omega \times Y \times Z)$. Since $p_{0} \geq 0$ and $\left(1-\theta_{0}\right) \geq 0$ a.e., we get

$$
p_{0}(x)\left(1-\theta_{0}(x, y, z)\right)=0, \quad \text { for a.e. }(x, y, z) \in \Omega \times Y \times Z \text {. }
$$

Actually, the result holds due to the multiplication of weakly and strongly converging sequences. Thus, we have the following:

(a) either $p_{0}>0$ and $\theta_{0}=1$,

(b) or $p_{0}=0$ and $0 \leq \theta_{0} \leq 1$,

so that $\theta_{0} \in H\left(p_{0}\right)$ a.e., which shows the thesis.

Lemma 3.3. The limit functions satisfy the following microdecompositions and macrodecomposition:

(1) macroscopic equation:

$$
\int_{\Omega}{\overline{h^{3}\left(\nabla p_{0}+\nabla_{y} p_{1}+\nabla_{z} p_{2}\right)}}^{Z \times Y} \nabla \phi=\int_{\Omega}{\overline{\theta_{0} h}}^{Z \times Y} \frac{\partial \phi}{\partial x_{1}}+\int_{\Gamma_{\star}} Q \phi, \quad \forall \phi \in V ;
$$

(2) microscopic equation at scale $\varepsilon$ : for a.e. $x \in \Omega$,

$$
\int_{Y}{\overline{h^{3}\left(\nabla p_{0}+\nabla_{y} p_{1}+\nabla_{z} p_{2}\right)}}^{Z} \nabla \psi=\int_{Y}{\overline{\theta_{0} h}}^{Z} \frac{\partial \psi}{\partial y_{1}}, \quad \forall \psi \in H_{\#}^{1}(Y) ;
$$

(3) microscopic equation at scale $\varepsilon^{2}$ : for a.e. $(x, y) \in \Omega \times Y$,

$$
\int_{Z} h^{3}\left(\nabla p_{0}+\nabla_{y} p_{1}+\nabla_{z} p_{2}\right) \nabla \varphi=\int_{Z} \theta_{0} h \frac{\partial \varphi}{\partial z_{1}}, \quad \forall \varphi \in H_{\#}^{1}(Z) .
$$

Here, ${ }^{-Y}$ (resp., ${ }^{-Z}$ ) denotes the averaged operator on $Y$ (resp., Z) with respect to $y$ (resp., $z$ ).

Proof. In the formulation of $\left(p_{\theta}^{\varepsilon}\right)$, let us consider a test function $\Phi$ defined by

$$
\Phi(x)=\phi^{(0)}(x)+\varepsilon \phi^{(1)}(x) \psi^{(1)}\left(\frac{x}{\varepsilon}\right)+\varepsilon^{2} \phi^{(2)}(x) \psi^{(2)}\left(\frac{x}{\varepsilon}\right) \varphi^{(2)}\left(\frac{x}{\varepsilon^{2}}\right),
$$

with $\phi^{(0)} \in V, \phi^{(i)} \in \Phi(\Omega), \psi^{(i)} \in H_{\#}^{1}(Y)(i \in\{1,2\}), \varphi^{(2)} \in H_{\#}^{1}(Z)$. Then, using the integration formula (see Proposition A.4), the limit in $\varepsilon$ yields the micro-/macrodecomposition.

Now, the goal is to get the homogenized equations, that is, only macroscopic equations describing the scale effects on the average flow. The general method relies on the possibility to solve local problems describing the coupling effects at the different scales. For this, we first introduce the local problems, whose structure will be justified in the proof of Lemma 3.6. 
Definition 3.4 (local problems at scale $\left.\varepsilon^{2}\right)$. Find $\mho^{(i)}(i=1,2), \alpha^{\star}, \alpha^{0}$ in $L^{2}\left(\Omega \times Y ; H_{\#}^{1}(Z) / \mathbb{R}\right)$, such that, for a.e. $(x, y) \in \Omega \times Y$ :

$$
\begin{gathered}
\int_{Z} h^{3} \nabla_{z} \mho^{(i)} \nabla_{z} \varphi=\int_{Z} h^{3} \frac{\partial \varphi}{\partial z_{i}}, \quad \forall \varphi \in H_{\#}^{1}(Z)(i=1,2), \\
\int_{Z} h^{3} \nabla_{z} \alpha^{\star} \nabla_{z} \varphi=\int_{Z} h \frac{\partial \varphi}{\partial z_{1}}, \quad \forall \varphi \in H_{\#}^{1}(Z), \\
\int_{Z} h^{3} \nabla_{z} \alpha^{0} \nabla_{z} \varphi=\int_{Z} \theta_{0} h \frac{\partial \varphi}{\partial z_{1}}, \quad \forall \varphi \in H_{\#}^{1}(Z) .
\end{gathered}
$$

Definition 3.5 (local problems at scale $\varepsilon$ ). Introduce the following coefficients:

$$
\begin{gathered}
\mathscr{L}^{(3)}={\overline{h^{3}\left(I-\nabla_{z} \mho\right)}}^{Z} \quad \text { with }=\left(\begin{array}{l}
\mho^{(1)} \\
\mho^{(2)}
\end{array}\right), \\
\mathscr{L}^{0}=\frac{\left(\begin{array}{c}
\theta_{0} h \\
0
\end{array}\right)-h^{3} \nabla_{z} \alpha^{0}}{Z}, \quad \mathscr{L}^{\star}=\frac{\left(\begin{array}{l}
h \\
0
\end{array}\right)-h^{3} \nabla_{z} \alpha^{\star}}{}
\end{gathered}
$$

Find $W^{(i)}(i=1,2), \beta^{\star}, \beta^{0}$ in $L^{2}\left(\Omega ; H_{\#}^{1}(Y) / \mathbb{R}\right)$, such that, for a.e. $x \in \Omega$,

$$
\begin{gathered}
\int_{Y} \mathscr{H}^{(3)} \cdot \nabla_{y} \mathcal{W}^{(i)} \nabla_{y} \psi=\sum_{k=1}^{2} \int_{Y} \mathscr{H}_{k i}^{(3)} \frac{\partial \psi}{\partial y_{k}}, \quad \forall \psi \in H_{\#}^{1}(Y)(i=1,2), \\
\int_{Y} \mathscr{H}^{(3)} \cdot \nabla_{y} \beta^{\star} \nabla_{y} \psi=\int_{Y} \mathscr{H}^{\star} \nabla_{y} \psi, \quad \forall \psi \in H_{\#}^{1}(Y), \\
\int_{Y} \mathscr{H}^{(3)} \cdot \nabla_{y} \beta^{0} \nabla_{y} \psi=\int_{Y} \mathscr{\ell}^{0} \nabla_{y} \psi, \quad \forall \psi \in H_{\#}^{1}(Y) .
\end{gathered}
$$

In a natural way, $\mathcal{W}$ will denote $\left(\begin{array}{c}w^{(1)} \\ w^{(2)}\end{array}\right)$.

\subsection{Homogenized problem: general case}

We first present the following partial homogenization result.

Lemma 3.6 (partial result in the general case). The main unknowns $\left(p_{0}, \theta_{0}\right) \in V \times L^{\infty}(\Omega \times Y \times Z)$ of the limit problem satisfy the following equations:

$$
\begin{gathered}
\int_{\Omega} \mathcal{A} \cdot \nabla p_{0} \nabla \phi=\int_{\Omega} B^{0} \nabla \phi+\int_{\Gamma_{\star}} Q \phi, \quad \forall \phi \in V, \\
p_{0} \geq 0, \quad \theta_{0} \in H\left(p_{0}\right) \quad \text { a.e., }
\end{gathered}
$$

with

$$
\begin{gathered}
\mathcal{A}={\overline{h^{3}\left(I-\nabla_{z} \mho\right)\left(I-\nabla_{y} \mathcal{W}\right)}}^{Z \times Y}, \\
B^{0}=\left(\begin{array}{c}
\theta_{0} h \\
0
\end{array}\right)-h^{3} \nabla_{z} \alpha^{0}-h^{3}\left(I-\nabla_{z} \mho\right) \cdot \nabla_{y} \beta^{0} \\
Z \times Y
\end{gathered}
$$


Remark 3.7. The proposed result is partial in the sense that it describes the coupling effects of both microscopic and macroscopic functions $\left(p_{0}\right.$ and $\left.\theta_{0}\right)$, instead of purely macroscopic functions ( $p_{0}$ and a macroscopic saturation function, e.g.). Still, as a first step, it allows to understand the structure of the limit problem.

Proof. The analysis first deals with the description of the interaction between the scale effects of order $\varepsilon^{2}$, on the one hand, and the scale effects of orders $\varepsilon$ and 1, on the other hand. From (3.8) and Definition 3.4 of the local problems and related solutions, we have

$$
p_{2}=-\mho\left(\nabla p_{0}+\nabla_{y} p_{1}\right)+\alpha^{0}, \quad \text { in } L^{2}\left(\Omega \times Y ; H_{\#}^{1}(Z) / \mathbb{R}\right),
$$

which describes the coupling of the different scales at the lowest scale $\varepsilon^{2}$.

Then, we deal with the description of the interaction between the scales of orders $\varepsilon$ and 1 (still taking into account the scale effects of order $\varepsilon^{2}$ ). For this, we insert (3.19) into (3.7) which leads, in a very natural way, to consider the local problems and related solutions described in Definition 3.5. Moreover, we obtain

$$
p_{1}=-\mathcal{W} \nabla p_{0}+\beta^{0}, \quad \text { in } L^{2}\left(\Omega ; H_{\#}^{1}(Y) / \mathbb{R}\right) .
$$

The last step describes the interaction of the scale effects of order $\varepsilon^{2}$ and $\varepsilon$ at the macroscopic level (scale of order 1). For this, we insert (3.19) and (3.20) into (3.6), which concludes the proof.

Theorem 3.8 (homogenized problem). One possible definition of the homogenized problem is

$$
\left(p_{\theta}^{\star}\right)\left\{\begin{array}{l}
f i n d\left(p_{0}, \Theta_{1}, \Theta_{2}\right) \in V \times L^{\infty}(\Omega) \times L^{\infty}(\Omega) \text { such that } \\
\int_{\Omega} \mathcal{A} \cdot \nabla p_{0} \nabla \phi=\int_{\Omega} \Theta_{i} B_{i}^{\star} \frac{\partial \phi}{\partial x_{i}}+\int_{\Gamma_{\star}} Q \phi, \quad \forall \phi \in V, \\
p_{0} \geq 0, \quad p_{0}\left(1-\Theta_{i}\right)=0,(i=1,2), \quad \text { a.e., }
\end{array}\right.
$$

where $\mathcal{A}$ is defined in Lemma 3.6 and $B^{\star}=\overline{\left(\begin{array}{c}h \\ 0\end{array}\right)-h^{3} \nabla_{z} \alpha^{\star}-h^{3}\left(I-\nabla_{z} \mho\right) \cdot \nabla_{y} \beta^{\star}}$ Z×Y

Proof. The result is a corollary of Lemma 3.6, in which the coefficients of the right-hand side have been renormalized as $\Theta_{i}=\mathbb{B}_{i}^{0} / \bar{B}_{i}^{\star}(i \in\{1,2\})$.

Thus,it appears that the homogenized problem $\left(D_{\theta}^{\star}\right)$ deals with saturation functions which may lack physical properties as we cannot guarantee that they are smaller than 1 in cavitated areas, nor can we treat two different saturation functions with a well-known numerical procedure. However, we show that it also admits a class of solutions which have physical relevance.

Theorem 3.9. The homogenized problem $\left(D_{\theta}^{\star}\right)$ admits at least one so-called isotropic solution $\left(p_{0}, \Theta, \Theta\right)$ with $\Theta \in H\left(p_{0}\right)$ a.e. 
Proof. Consider the penalized version of the following problem:

$$
\left(D_{\eta}^{\varepsilon}\right)\left\{\begin{array}{l}
\text { find } p_{\eta, \varepsilon} \in V, \text { such that } \\
\int_{\Omega} h_{\varepsilon}^{3} \nabla p_{\eta, \varepsilon} \nabla \phi=\int_{\Omega} H_{\eta}\left(p_{\eta, \varepsilon}\right) h_{\varepsilon} \frac{\partial \phi}{\partial x_{1}}+\int_{\Gamma_{\star}} Q \phi, \quad \forall \phi \in V, \\
p_{\eta, \varepsilon} \geq 0, \quad \text { a.e., }
\end{array}\right.
$$

where $H_{\eta}(z)=(z / \eta) \mathbf{1}_{[0, \eta[}+\mathbf{1}_{[\eta,+\infty[}$ mimics the Heaviside graph. It can be proved (see [10-12]) that $\left(D_{\eta}^{\varepsilon}\right)$ admits a unique solution. Using the same steps as before, the homogenization of this penalized problem leads to the following asymptotic problem:

$$
\left(D_{\eta}^{\star}\right)\left\{\begin{array}{l}
\text { find } p_{0}^{\eta} \in V \text { such that } \\
\int_{\Omega} \mathcal{A} \cdot \nabla p_{0}^{\eta} \nabla \phi=\int_{\Omega} H_{\eta}\left(p_{0}^{\eta}\right) B^{\star} \nabla \phi+\int_{\Gamma_{\star}} Q \phi, \quad \forall \phi \in V, \\
p_{0}^{\eta} \geq 0, \quad \text { a.e. }
\end{array}\right.
$$

The proof is concluded by passing to the limit on $\eta$.

Remark 3.10. The difficulties that we have mentioned are related to the ones addressed in $[16,17]$ for the dam problem (whose mathematical structure is very close to the lubrication problem). On the one hand, the right-hand side of the homogenized problem $\mathbb{B}^{0}$ leads to anisotropic effects on the saturation (see Lemma 3.6 and Theorem 3.8), which lacks physical simple interpretation. On the other hand, we have been able to build an isotropic solution to this problem, with more physical properties on the saturation. If $\left(p_{0}, \Theta, \Theta\right)$ denotes an isotropic solution of the homogenized problem, one cannot, in general, have the convergence of $\theta_{\varepsilon}$ to $\Theta$ (see the counter example of Rodrigues [17] for the dam problem, which can be adapted to the lubrication problem), and the issue of relating $\mathbb{B}^{0}$ (which highly depends on $\theta_{0}$ ) to $\Theta \mathbb{B}^{\star}$ is not clear.

In fact, as will be seen further, it is possible to get rid of the mentioned difficulties, under some additional assumptions on the roughness patterns.

\subsection{Homogenized problem: particular cases}

Here, we prove that under some assumptions on the roughness patterns, the homogenized problem is well-posed from both mathematical and physical points of view.

Definition 3.11. Denote $h_{[i j]}(x, y, z):=h\left(x, y_{i}, z_{j}\right)$, that is, $h_{[i j]}$ only depends on $x, y_{i}$, and $z_{j}$ $(i, j \in\{1,2\})$.

Notice that although it prevents us from describing all the two-dimensional defaults, roughness patterns described by Definition 3.11 fall into the scope of typical defaults observed in many applications. Indeed, the manufacturing processes often involve a tooling of the cylinders following orthogonal directions (namely, $x_{1}$ and $x_{2}$ ), thus leading to roughness defaults in the machining directions. Thus, gap functions described by Definition 3.11 are realistic in many applications. 
Table 1: Homogenized coefficients and link between the micro/ macrosaturations.

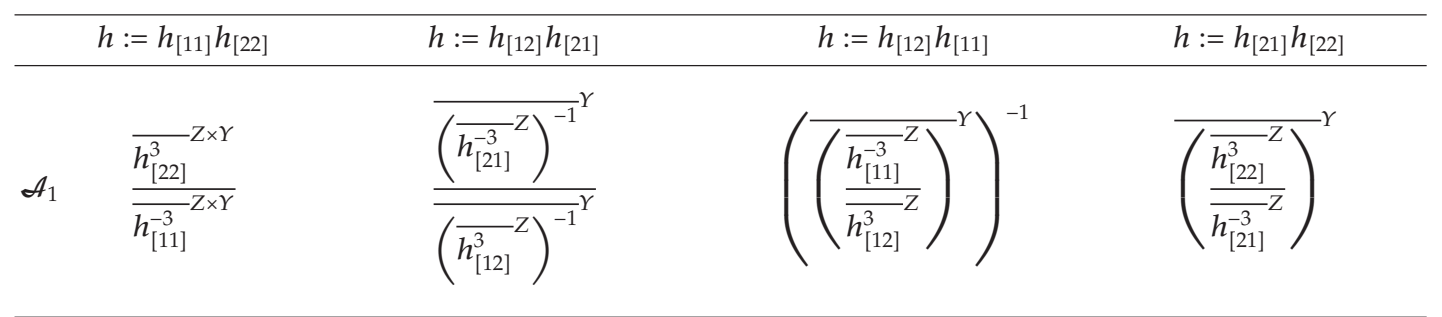

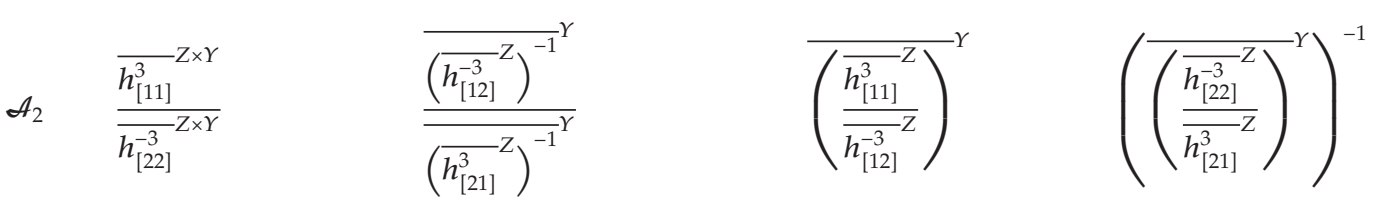

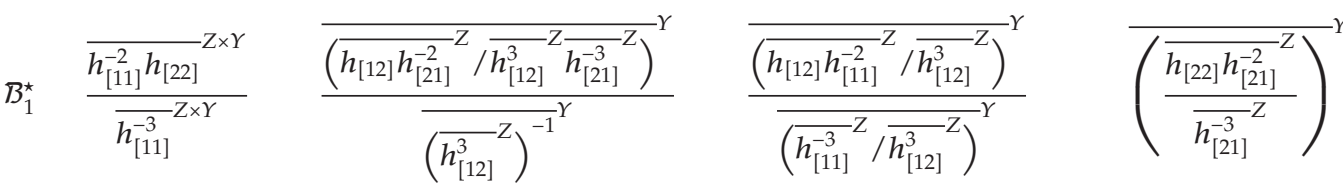

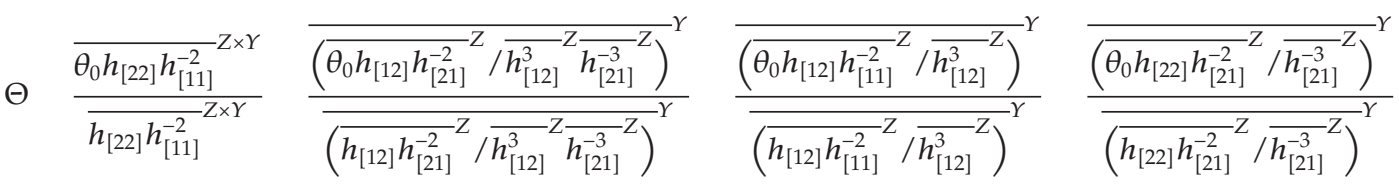

Theorem 3.12. Let $(i, j) \in\{1,2\}, i \neq j$. If $h$ can be identified to a function $h_{[i j]}, h_{[i i]}, h_{[i j]} h_{[i i]}$, or $h_{[j i]} h_{[i i]}$, then the homogenized problem is

$$
\left(D_{\theta}^{\star}\right)\left\{\begin{array}{l}
\text { find }\left(p_{0}, \Theta\right) \in V \times L^{\infty}(\Omega) \text { such that } \\
\int_{\Omega}\left(\begin{array}{cc}
A_{1} & 0 \\
0 & \mathcal{A}_{2}
\end{array}\right) \cdot \nabla p_{0} \nabla \phi=\int_{\Omega} \Theta 乃_{1}^{\star} \frac{\partial \phi}{\partial x_{1}}+\int_{\Gamma_{\star}} Q \phi, \quad \forall \phi \in V, \\
p_{0} \geq 0, \quad \Theta \in H\left(p_{0}\right), \text { a.e., }
\end{array}\right.
$$

the homogenized coefficients being given by Table 1. The link between the microsaturation $\theta_{0}$ and the macro- (homogenized) saturation $\Theta$ is also provided by Table 1. Moreover, $\left(D_{\theta}^{\star}\right)$ admits a unique solution.

Proof. Assumptions on the roughness patterns lead to some particular anisotropy of the scale effects. It allows us to solve explicitly the local problems by means of integration, using the separation of microvariables at the different scales. Technical computations are made explicit in the appendices, for the case $h:=h_{[12]}$, and may be easily adapted to the other situations (computations are omitted for convenience). 


\section{A numerical simulation}

In this section, the numerical simulation of a hydrodynamic contact is performed to illustrate the theoretical convergence results proved in the previous section. To this aim, we use the Bermudez-Moreno algorithm coupled to a characteristics' method, the combination of these numerical techniques being proved to be rigorous and efficient (see $[14,18]$ ). In particular, the basic principles of the algorithm (and related proofs of convergence) may be found in [14] for the lubrication problem.

We address the numerical simulation of dimensionless journal bearing contacts so that, for a domain $\Omega=] 0,1[\times] 0,1$, problem $\left(p_{\theta}^{\varepsilon}\right)$ is considered. The datum $h_{\varepsilon}$ is given by

$$
h_{\varepsilon}(x)=1+\rho \cos \left(2 \pi x_{1}\right)+0.35(1-\rho) \sin \left(2 \pi \frac{x_{1}}{\varepsilon^{2}}\right)+0.35(1-\rho) \sin \left(2 \pi \frac{x_{2}}{\varepsilon}\right)
$$

where $\rho$ denotes the average eccentricity of the device.

Additionally, the input flow $Q$ has been taken to

$$
Q=\theta_{\star}(1+\rho),
$$

where $\theta_{\star}$ denotes the saturation at the supply groove. In the numerical tests, the following values have been considered:

$$
\rho=0.5, \quad \theta_{\star}=0.375
$$

Notice that the corresponding gap $h$ only depends on the variables $x, y_{2}$, and $z_{1}$. As a consequence, it can be identified to some function $h_{[21]}$ (see Definition 3.11), which satisfies the hypotheses of Theorem 3.12. Corresponding homogenized coefficients are provided by Table 1 and may be easily computed.

Although numerical tests have been performed for different spatial meshes in order to control the convergence of the method, we just present the results corresponding to a mesh size $900 \times 100$. Computations have been made for different values of $\varepsilon$ (namely, 1/4, 1/6, $1 / 8$ ) and for the corresponding homogenized case. The numerical experiments illustrate the convergence results proved in the previous sections.

(A) Figures 4-6 show the pressure (left) and saturation (right) distribution in different cases as follows:

(i) Figure 4: $\varepsilon=1 / 4$;

(ii) Figure 5: $\varepsilon=1 / 6$;

(iii) Figure 6: homogenized case.

In particular, oscillatory effects induced by the roughness patterns may be easily observed.

(B) As the introduction of the oscillating gap $h_{\varepsilon}$ leads to oscillatory effects in both transverse and longitudinal directions, we study some particular curves at different sections in order to observe the following oscillations: 


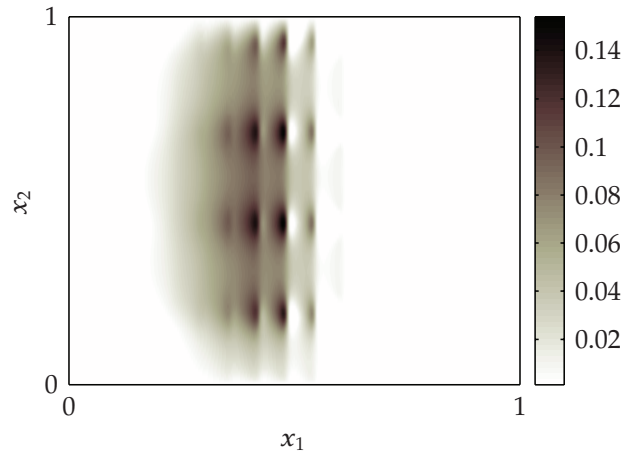

(a)

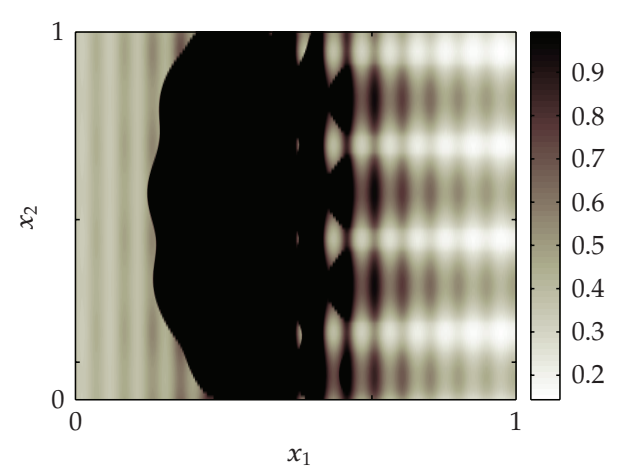

(b)

Figure 4: Pressure (a) and saturation (b) in the whole domain for $\varepsilon=1 / 4$.

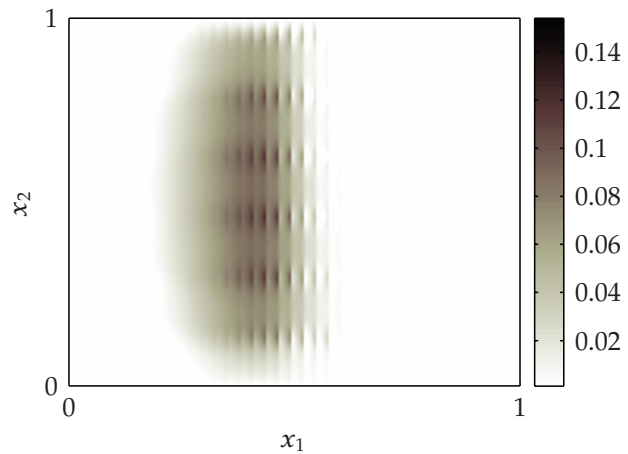

(a)

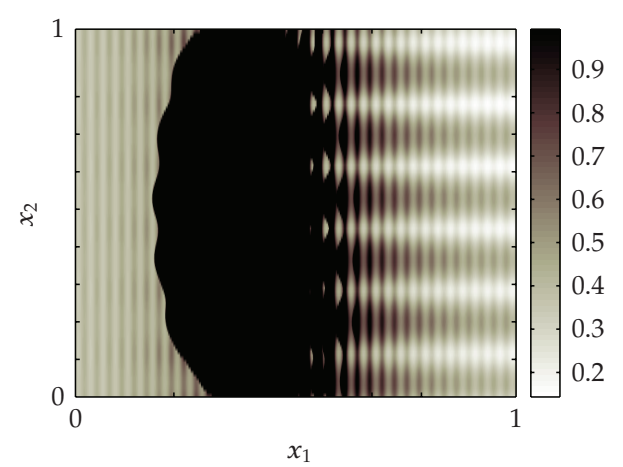

(b)

Figure 5: Pressure (a) and saturation (b) in the whole domain for $\varepsilon=1 / 6$.

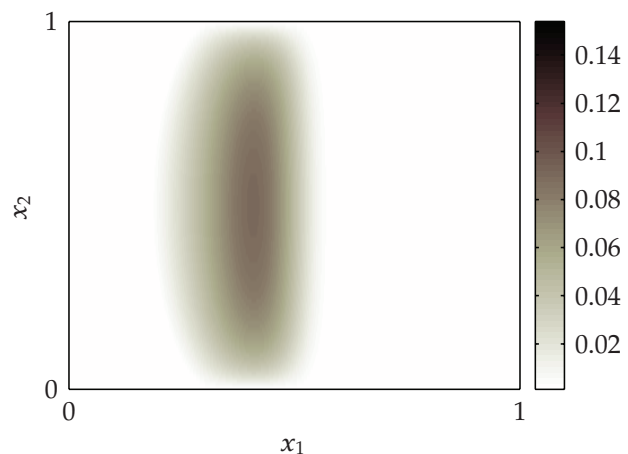

(a)

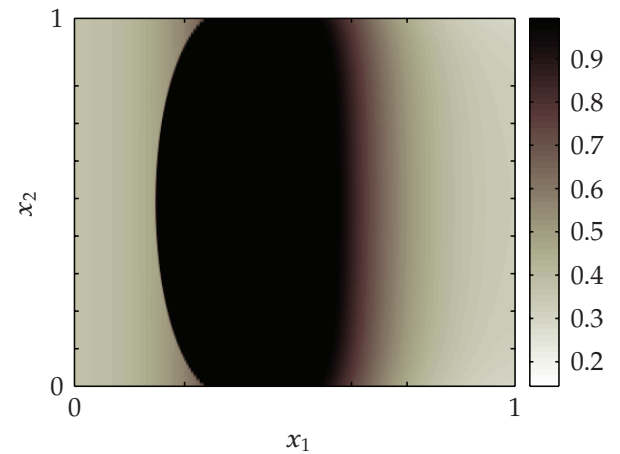

(b)

Figure 6: Homogenized pressure (a) and saturation (b) in the whole domain. 


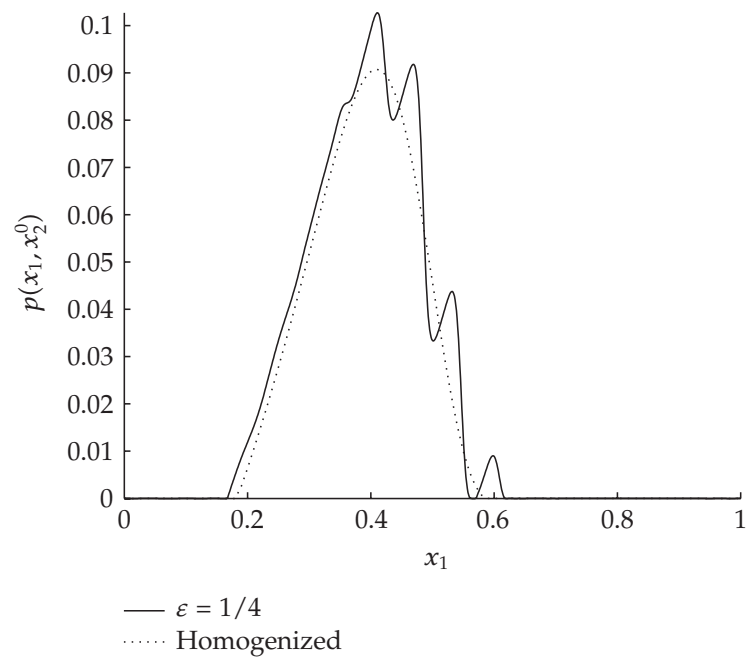

Figure 7: Pressure distribution at fixed $x_{2}^{0}=0.5$.

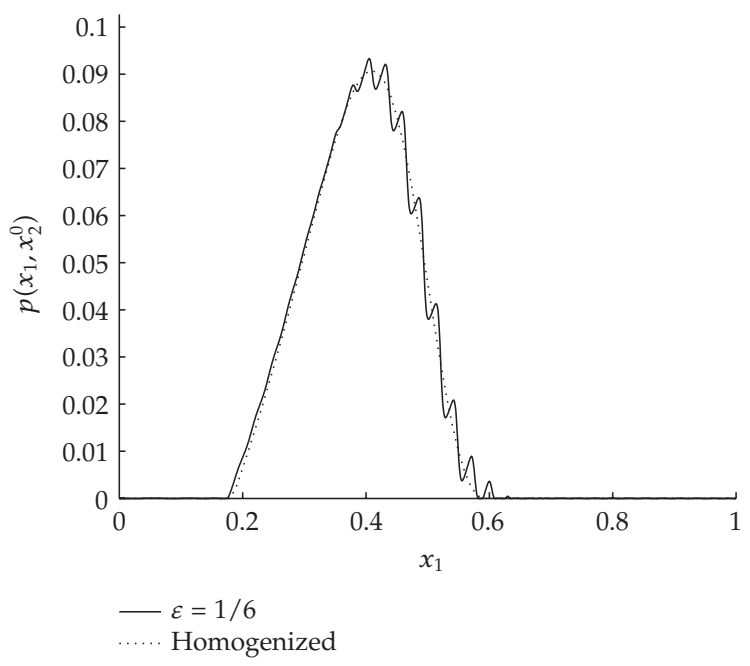

Figure 8: Pressure distribution at fixed $x_{2}^{0}=0.5$.

(1) Figures 7 and 8 (resp., Figures 9 and 10) correspond to pressure (saturation) plots at $x_{2}^{0}=0.5$ (midsection containing the homogenized peak pressure, for geometrical reasons). We show the convergence of the pressure to the homogenized (smooth) one, as $\varepsilon$ tends to 0 . Unlike the behavior of the pressure, the behavior of the saturation is more complicated. Oscillations are not damped, thus illustrating the weak convergence of the saturation. However, this does not prevent us from defining an equivalent homogenized saturation.

(2) Figures 11 and 12 correspond to pressure plots at $x_{1}^{0}=0.4118$ (section containing the homogenized peak pressure). The convergence of the pressure to the homogenized (smooth) 


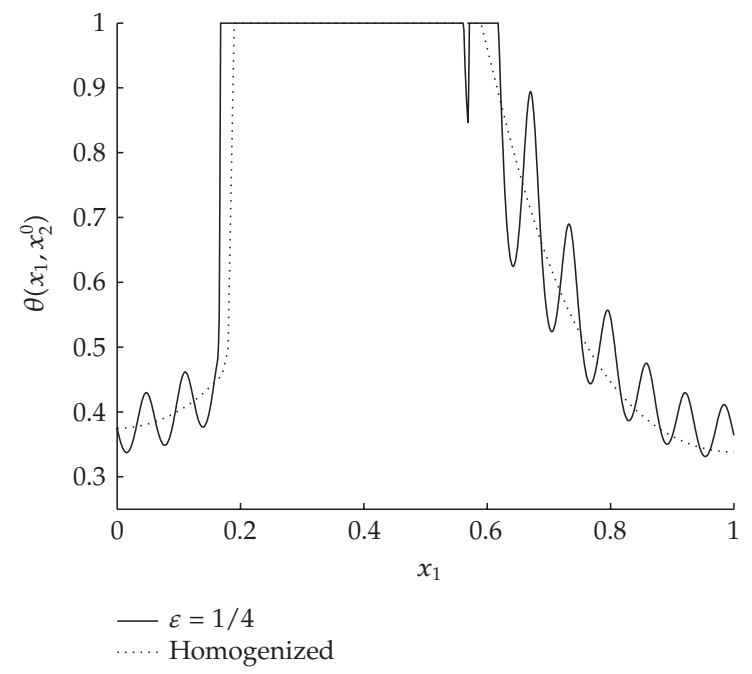

Figure 9: Pressure distribution at fixed $x_{2}^{0}=0.5$.

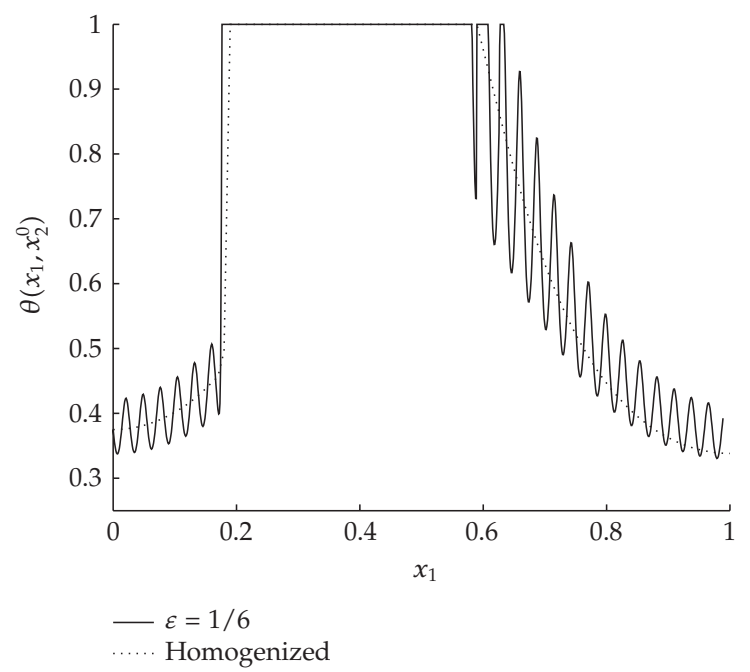

Figure 10: Saturation distribution at fixed $x_{2}^{0}=0.5$.

one is also illustrated. Corresponding saturation curves are omitted (since no cavitation appears in this section).

Thus, the convergence of the solution to the homogenized solution, with respect to the roughness parameter $\varepsilon$, is illustrated in this section. In particular, the asymptotic study allows us not only to determine the effective pressure but also to highlight the anisotropic effects on the saturation. Although highly oscillating in cavitated areas, an effective saturation weighting the roughness effects in each direction can be computed. 


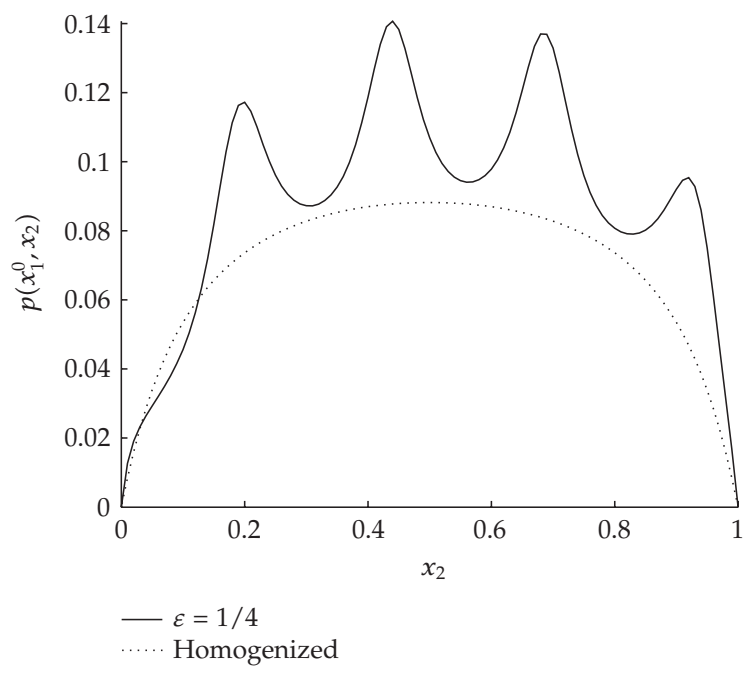

Figure 11: Saturation distribution at fixed $x_{1}^{0}=0.4118$.

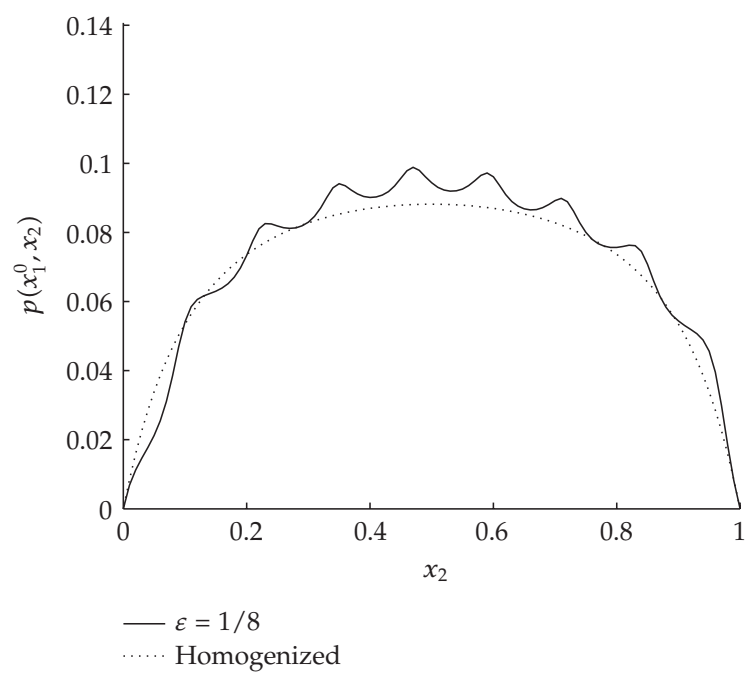

Figure 12: Saturation distribution at fixed $x_{1}^{0}=0.4118$.

\section{Conclusion}

The influence of roughness patterns on a thin film flow in a journal bearing has been investigated in this paper. In the most general case (i.e., without any assumption on the roughness geometry), a so-called "isotropic" asymptotic solution can be computed. Moreover, under specific additional assumptions which are realistic in terms of mechanical applications, 
the limit problem is well-posed, and anisotropic effects on the asymptotic flow are fully detailed, in particular, in cavitated areas.

The use of abrasive grains to form a surface in manufacturing processes may lead to different default scales $\varepsilon, \varepsilon^{2}, \varepsilon^{3}$, and so forth, which can be taken into account without additional theoretical difficulty. A computational procedure in order to derive the homogenized coefficients can be used from the smallest scale to the macroscopic one, by using successive solutions of local problems.

\section{Appendices}

\section{A. The periodic unfolding method}

The periodic unfolding method has been introduced by Cioranescu et al. [19]. It combines a dilatation technique, which was used by Arbogast et al. [20], and averaging approximations, thus reducing the asymptotic analysis to the study of weak convergences in appropriate spaces. This mathematical tool, which applies to multiscale problems in a very simple way, has strong links with the multiscale convergence technique introduced by Nguetseng [21], and further developed by Allaire [22], Allaire and Briane [23], and Lukkassen et al. [24].

Let $\Omega$ be an open-bounded subset of $\mathbb{R}^{d}, d \in \mathbb{N}^{\star}$, and let $\left.Y=\right] 0,1\left[{ }^{d}\right.$ denote the reference cell (eventually, $Z$ will also denote the reference cell). Then, for any $x \in \mathbb{R}^{d},[x]_{Y} \in \mathbb{Z}^{d}$ denotes the unique element such that $x-[x]_{Y}$ belongs to $Y$.

Definition A.1. Let $\widetilde{\Omega}_{n}=\Omega \times Y^{n}, n \in \mathbb{N}$, with $\widetilde{\Omega}_{0}=\Omega$. The unfolding operator

$$
\begin{aligned}
& \tau_{\varepsilon}: L^{2}\left(\widetilde{\Omega}_{n}\right) \longrightarrow L^{2}\left(\widetilde{\Omega}_{n+1}\right) \\
& w \longrightarrow \tau_{\varepsilon}(w)
\end{aligned}
$$

modifies any function $w \in L^{2}\left(\widetilde{\Omega}_{n}\right)$, extended by 0 outside $\widetilde{\Omega}_{n}$, as follows:

(a) if $n=0, \tau_{\varepsilon}(w)(x, y)=w\left(\varepsilon[x / \varepsilon]_{Y}+\varepsilon y\right)$;

(b) if $n \geq 1, \tau_{\varepsilon}(w)\left(x, y^{(1)}, \ldots, y^{(n+1)}\right)=w\left(x, y^{(1)}, \ldots, y^{(n-1)},\left[y^{(n)} / \varepsilon\right]_{Y}+\varepsilon y^{(n+1)}\right)$.

This definition leads, in a natural way, to reiterated unfolding operators (of any order $k \in \mathbb{N}^{\star}$ )

$$
\tau_{\delta_{k} \circ \delta_{k-1} \circ \cdots \circ \delta_{1}}: L^{2}\left(\widetilde{\Omega}_{n}\right) \longrightarrow L^{2}\left(\widetilde{\Omega}_{n+k}\right)
$$

defined by

$$
\tau_{\delta_{k} \circ \delta_{k-1} \circ \cdots \circ \delta_{1}}=\tau_{\delta_{k}} \circ \tau_{\delta_{k-1}} \circ \cdots \circ \tau_{\delta_{1}}
$$

Example A.2. Let us consider some function $f \in L^{2}\left(\Omega ; C_{\#}^{1}(Y \times Y)\right)$ and define $f_{\delta \varepsilon}$ by

$$
f_{\delta \varepsilon}(x)=f\left(x, \frac{x}{\varepsilon}, \frac{x}{\delta \varepsilon}\right)
$$

Then, we may observe the following. 
(a) The unfolding operator $\tau_{\varepsilon}: L^{2}(\Omega) \rightarrow L^{2}(\Omega \times Y)$ does not see the oscillations at scale $\delta \varepsilon$. Indeed,

$$
\tau_{\varepsilon}\left(f_{\delta \varepsilon}\right)(x, y)=f\left(x, y, \frac{y}{\delta}\right)
$$

which does not outline the oscillating periods induced by the parameter $\delta$.

(b) The reiterated unfolding operator $\tau_{\delta \circ \varepsilon}: L^{2}(\Omega) \rightarrow L^{2}(\Omega \times Y \times Z)$ allows to capture the oscillatory effects at both scales $\varepsilon$ and $\delta \varepsilon$. Indeed,

$$
\tau_{\delta \circ \varepsilon}\left(f_{\delta \varepsilon}\right)(x, y, z)=f_{\delta \varepsilon}\left(\varepsilon\left[\frac{x}{\varepsilon}\right]_{Y}+\varepsilon \delta\left[\frac{y}{\delta}\right]_{Z}+\varepsilon \delta z\right)=f(x, y, z),
$$

leading to an effective (but artificial) separation of the scale effects.

Proposition A.3 (see [19]). (i) Let $u_{\varepsilon}$ be a bounded sequence in $L^{2}(\Omega)$. Then, there exists $u_{0} \in$ $L^{2}(\Omega \times Y)$ such that, up to a subsequence,

$$
\tau_{\varepsilon}\left(u_{\varepsilon}\right) \rightarrow u_{0}, \quad \text { in } L^{2}(\Omega) .
$$

(ii) Let $u_{\varepsilon}$ be a bounded sequence in $H^{1}(\Omega)$, which weakly converges to a limit $u_{0} \in H^{1}(\Omega)$. Then, with $\tau_{\varepsilon \circ \ldots \circ \varepsilon}$ denoting the reiterated unfolding operator of order $k \in \mathbb{N}^{\star}$, one has, up to an extraction,

$$
\tau_{\varepsilon \circ \cdots \circ \varepsilon}\left(u_{\varepsilon}\right) \longrightarrow u_{0}, \quad \text { in } L^{2}\left(\tilde{\Omega}_{k}\right),
$$

and there exist functions $u_{i} \in L^{2}\left(\widetilde{\Omega}_{i-1} ; H_{\#}^{1}(Y) / \mathbb{R}\right)(i \in\{1, \ldots, k\})$, such that

$$
\tau_{\varepsilon \circ \cdots \varepsilon \varepsilon}\left(\nabla u_{\varepsilon}\right) \rightarrow \nabla u_{0}+\sum_{i=1}^{k} \nabla_{y^{(i)}} u_{i} \quad \text { in } L^{2}\left(\widetilde{\Omega}_{k}\right)
$$

Proposition A.4 (see [19]). One has the following integration formulas:

$$
\int_{\tilde{\Omega}_{n}} w=\int_{\tilde{\Omega}_{n+1}} \tau_{\delta_{1}}(w)=\cdots=\int_{\tilde{\Omega}_{n+k}} \tau_{\delta_{k} \circ \cdots \circ \delta_{1}}(w), \quad \forall w \in L^{1}\left(\tilde{\Omega}_{n}\right) .
$$

\section{B. Computation of the homogenized coefficients}

Let us assume that $h:=h_{[12]}$ meaning that $h$ only depends on $x, y_{1}$, and $z_{2}$. We compute the homogenized coefficients under this specific assumption. Let us recall the way these effective coefficients describing the average flow are defined.

(a) We first introduce the coefficients averaged with respect to the $z$ variable (lowest scale):

$$
\begin{aligned}
\mathscr{H}^{(3)} & :={\overline{h^{3}\left(I-\nabla_{z} \mathcal{U}\right)}}^{Z}, \\
\mathscr{H}^{0} & :={\overline{\left(\begin{array}{c}
\theta_{0} h \\
0
\end{array}\right)-h^{3} \nabla_{z} \alpha^{0},}}^{Z}, \\
\mathscr{H}^{\star} & :={\overline{\left(\begin{array}{c}
h \\
0
\end{array}\right)-h^{3} \nabla_{z} \alpha^{\star},}}^{Z},
\end{aligned}
$$

where $\mho^{(i)}, \alpha^{0}$, and $\alpha^{\star}$ are defined by (3.10)-(3.12). 
(b) Then we introduce the coefficients averaged with respect to the $y$ variable (intermediary scale):

$$
\begin{aligned}
& \mathcal{A}:={\overline{h^{3}\left(I-\nabla_{z} \mho\right)\left(I-\nabla_{y} \mathcal{W}\right)}}^{Z \times Y}={\overline{\mathscr{L}^{(3)}\left(I-\nabla_{y} \mathcal{W}\right)}}^{Y} \text {, } \\
& B^{0}:=\overline{\left(\begin{array}{c}
\theta_{0} h \\
0
\end{array}\right)-h^{3} \nabla_{z} \alpha^{0}-h^{3}\left(I-\nabla_{z} \mho\right) \cdot \nabla_{y} \beta^{0}}=\overline{\mathscr{L}^{0}-\mathscr{L}^{(3) \cdot \nabla_{y} \beta^{0}}} \Upsilon \text {, } \\
& B^{\star}:=\overline{\left(\begin{array}{l}
h \\
0
\end{array}\right)-h^{3} \nabla_{z} \alpha^{\star}-h^{3}\left(I-\nabla_{z} \mho\right) \cdot \nabla_{y} \beta^{\star}}=\overline{\mathscr{L}^{\star}-\mathscr{L}^{(3)} \cdot \nabla_{y} \beta^{\star}} \text {, }
\end{aligned}
$$

where $W^{(i)}, \beta^{0}$, and $\beta^{\star}$ are defined by (3.14)-(3.16).

Now let us compute the following coefficients.

Step 1 (computation of the matrix $\left.\mathscr{H}^{(3)}:={\overline{h^{3}\left(I-\nabla_{z} \mathcal{V}\right)}}^{Z}\right)$. By definition, $\mho^{(i)}(i=1,2)$ satisfies, for a.e. $(x, y) \in \Omega \times Y$,

$$
\int_{Z} h^{3} \nabla_{z} \mho^{(i)} \nabla_{z} \varphi=\int_{Z} h^{3} \frac{\partial \varphi}{\partial z_{i}}, \quad \forall \varphi \in H_{\#}^{1}(Z)(i=1,2) .
$$

If we use a test function only depending on $z_{i}$ or $z_{j}$ (by convention, $j \neq i$ ), we get

$$
\begin{gathered}
\int_{Z_{i}} \overline{h^{3} \frac{\partial \mho^{(i)}}{\partial z_{i}}} \frac{Z_{j}}{d z_{i}}=\int_{Z_{i}} \overline{h^{3}} Z_{j} \frac{d \varphi}{d z_{i}}, \quad \forall \varphi \in H_{\#}^{1}\left(Z_{i}\right), \\
\int_{Z_{j}} \overline{h^{3} \frac{\partial \mho^{(i)}}{\partial z_{j}}} \frac{Z_{i}}{d z_{j}}=0, \quad \forall \varphi \in H_{\#}^{1}\left(Z_{j}\right) .
\end{gathered}
$$

These two equations lead to the following equalities, which hold for a.e. $(x, y) \in \Omega \times Y$ :

$$
\begin{gathered}
\overline{h_{[12]}^{3} \frac{\partial \mho^{(i)}}{\partial z_{i}} Z_{j}}={\overline{h_{[12]}^{3}}}^{Z_{j}}-C_{x, y}, \\
\frac{h_{[12]}^{3} \frac{\partial \mho^{(i)}}{\partial z_{j}}}{Z_{i}}=-C_{x, y},
\end{gathered}
$$

where $C_{x, y}$ denotes any constant with respect to $z$. Now, let us analyze each equality.

(i) Equality (B.5) with $(i, j)=(1,2)$. We average the equality with respect to $z_{1}$ so that

$$
{\overline{h_{[12]}^{3}}}^{Z}-C_{x, y}^{(1)}={\overline{h^{3} \frac{\partial \mho^{(1)}}{\partial z_{1}}}}^{Z}=\bar{h}_{[12]}^{3{\overline{\frac{\partial \mho^{(1)}}{\partial z_{1}}}}^{Z} Z^{Z_{2}}}=0,
$$

due to the periodicity of $\mho^{(1)}$ in the $z_{1}$ variable. We thus obtain $C_{x, y}^{(1)}={\overline{h_{[12]}^{3}}}^{Z}$. 
(ii) Equality (B.5) with $(i, j)=(2,1)$. Dividing the equality by $h_{[12]}^{3}$ (which does not depend on $z_{1}$ ), we get

$$
{\frac{\partial \mathcal{U}^{(2)}}{\partial z_{2}}}^{Z_{1}}=1-C_{x, y}^{(2)} h_{[12]}^{-3}
$$

Integrating with respect to $z_{2}$, we get

$$
1-C_{x, y}^{(2)}{\frac{h_{[12]}^{-3}}{Z}}^{Z}=\frac{{\overline{\partial \mho^{(2)}}}^{Z}}{\partial z_{2}}=0,
$$

due to the periodicity of $\mho^{(2)}$ in the $z_{2}$ variable. Thus,

$$
C_{x, y}^{(2)}=\left({\overline{h_{[12]}^{-3}}}^{Z}\right)^{-1}
$$

(iii) Equality (B.6) with $(i, j)=(1,2)$. Dividing the equality by $h_{[12]}^{3}$ (which does not depend on $z_{1}$ ) and integrating with respect to $z_{2}$, we get

$$
-C_{x, y}^{(3)}{\overline{h_{[12]}^{-3}}}^{Z_{2}}=\frac{{\overline{\partial \mho^{(1)}}}^{Z}}{\partial z_{2}}=0
$$

due to the periodicity of $\mho^{(1)}$ in the $z_{2}$ variable. We conclude that $C_{x, y}^{(3)}=0$.

(iv) Equality (B.6) with $(i, j)=(2,1)$. Integrating the equality with respect to $z_{1}$, we get

$$
-C_{x, y}^{(4)}={\overline{h_{[12]}^{3} \frac{\partial \mho^{(2)}}{\partial z_{1}}}}^{Z}=\bar{h}_{[12]}^{3{\overline{\frac{\partial \mho^{(2)}}{\partial z_{1}}}}^{Z_{1}}}=0,
$$

due to the periodicity of $\mho^{(2)}$ in the $z_{1}$ variable. We conclude that $C_{x, y}^{(4)}=0$.

The coefficients $C_{x, y}^{(i)}$ exactly define the matrix $\mathscr{L}^{(3)}$ and we have

$$
\mathscr{H}^{(3)}:=\left(\begin{array}{ll}
C_{x, y}^{(1)} & C_{x, y}^{(4)} \\
C_{x, y}^{(3)} & C_{x, y}^{(2)}
\end{array}\right)=\left(\begin{array}{cc}
\overline{h_{[12]}^{3}} & 0 \\
0 & \left(\frac{\bar{h}_{[12]}^{-3}}{Z}\right)^{-1}
\end{array}\right) .
$$

Step 2 (computation of the vector $\mathscr{\ell}^{0}=\overline{\left(\begin{array}{c}\theta_{0} h \\ 0\end{array}\right)-h^{3} \nabla_{z} \alpha^{0}}$ Z ). By definition, $\alpha^{0}$ satisfies, for a.e. $(x, y) \in \Omega \times Y$,

$$
\int_{Z} h^{3} \nabla_{z} \alpha^{0} \nabla_{z} \varphi=\int_{Z} \theta_{0} h \frac{\partial \varphi}{\partial z_{1}}, \quad \forall \varphi \in H_{\#}^{1}(Z)
$$


If we use a test function only depending on $z_{1}$ or $z_{2}$, we get

$$
\begin{gathered}
\int_{Z_{1}} \overline{h^{3} \frac{\partial \alpha^{0}}{\partial z_{1}}} \frac{d \varphi}{d z_{1}}=\int_{Z_{1}}{\overline{\theta_{0} h}}^{Z_{2}} \frac{d \varphi}{d z_{1}}, \quad \forall \varphi \in H_{\#}^{1}\left(Z_{1}\right), \\
\int_{Z_{2}} \overline{h^{3} \frac{\partial \alpha^{0}}{\partial z_{2}}} \frac{d \varphi}{d z_{2}}=0, \quad \forall \varphi \in H_{\#}^{1}\left(Z_{2}\right) .
\end{gathered}
$$

These two equations lead to the following equalities, which hold for a.e. $(x, y) \in \Omega \times Y$ :

$$
\begin{gathered}
\overline{h_{[12]}^{3} \frac{\partial \alpha^{0}}{\partial z_{1}}} Z_{2}={\overline{\theta_{0} h_{[12]}}}^{Z_{2}}-D_{x, y}, \\
\frac{h_{[12]}^{3} \frac{\partial \alpha^{0}}{\partial z_{2}}}{Z_{1}}=-D_{x, y},
\end{gathered}
$$

where $D_{x, y}$ denotes any constant with respect to $z$. Now, let us analyze each equality.

(a) Equality (B.16). We average the equality with respect to $z_{1}$ so that

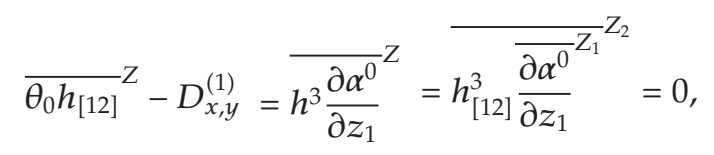

due to the periodicity of $\alpha^{0}$ in the $z_{1}$ variable. We thus obtain $D_{x, y}^{(1)}={\overline{\theta_{0} h_{[12]}}}^{Z}$.

(b) Equality (B.17). Dividing the equality by $h_{[12]}^{3}$ and integrating with respect to $z_{2}$, we get

$$
-D_{x, y}^{(2)}{\overline{h_{[12]}^{-3}}}^{Z_{2}}=\frac{{\overline{\partial \alpha^{0}}}^{Z}}{\partial z_{2}}=0
$$

due to the periodicity of $\alpha^{0}$ in the $z_{2}$ variable. We conclude that $D_{x, y}^{(2)}=0$.

The coefficients $D_{x, y}^{(i)}$ exactly define the vector $\mathscr{L}^{0}$ as

$$
\mathscr{L}^{0}:=\left(\begin{array}{c}
D_{x, y}^{(1)} \\
D_{x, y}^{(2)}
\end{array}\right)=\left(\begin{array}{c}
\overline{\theta_{0} h_{[12]}} \\
0
\end{array}\right)
$$

Step 3 (computation of the vector $\mathscr{L}^{\star}=\overline{\left(\begin{array}{l}h \\ 0\end{array}\right)-h^{3} \nabla_{z} \alpha^{\star}}$ ). The procedure follows the one explained in Step 2 (replacing $\theta_{0}$ by 1 and $\alpha^{0}$ by $\alpha^{\star}$ ). We obtain

$$
\mathscr{L}^{\star}:=\left(\begin{array}{c}
{\overline{h_{[12]}}}^{Z} \\
0
\end{array}\right) \text {. }
$$


Step 4 (computation of the matrix $\mathscr{A}:={\overline{\mathscr{L}^{(3)}\left(I-\nabla_{y} \mathcal{W}\right)}}^{\text {}}$ ). Let us recall that, by definition, $W^{(i)}(i=1,2)$ satisfies, for a.e. $x \in \Omega$,

$$
\int_{Y} \mathscr{H}^{(3)} \cdot \nabla_{y} \mathcal{W}^{(i)} \nabla_{y} \psi=\sum_{k=1}^{2} \int_{Y} \mathscr{H}_{k i}^{(3)} \frac{\partial \psi}{\partial y_{k}}, \quad \forall \psi \in H_{\#}^{1}(Y)(i=1,2)
$$

Due to the simplifications based on the particular choice for $h$, we obtain

$$
\int_{Y} \mathscr{L}^{(3)} \cdot \nabla_{y} \mathcal{W}^{(i)} \nabla_{y} \psi=\int_{Y} \mathscr{L}_{i i}^{(3)} \frac{\partial \psi}{\partial y_{i}}, \quad \forall \psi \in H_{\#}^{1}(Y)(i=1,2) .
$$

If we use a test function only depending on $y_{i}$ or $y_{j}$, we get

$$
\begin{gathered}
\int_{Y_{i}} \overline{\mathscr{H}_{i i}^{(3)}} \frac{\partial \mathcal{W}^{(i)}}{\partial y_{i}} \frac{Y_{j}}{d y_{i}}=\int_{Y_{i}} \overline{\mathcal{H}_{i i}^{(3)}} \frac{Y_{j}}{d \psi}, \quad \forall \psi \in H_{\#}^{1}\left(Y_{i}\right), \\
\int_{Y_{j}} \overline{\mathscr{H}_{j j}^{(3)} \frac{\partial \mathcal{W}^{(i)}}{\partial y_{j}}} \frac{Y_{i}}{d y_{j}}=0, \quad \forall \psi \in H_{\#}^{1}\left(Y_{j}\right) .
\end{gathered}
$$

These two equations lead to the following equalities, which hold for a.e. $x \in \Omega$ :

$$
\begin{gathered}
\overline{\mathscr{H}_{i i}^{(3)} \frac{\partial \mathcal{W}^{(i)}}{\partial y_{i}}} Y_{j}={\overline{\mathcal{H}_{i i}^{(3)}}}^{Y_{j}}-C_{x} \\
\frac{\mathscr{\ell}_{j j}^{(3)} \frac{\partial \mathcal{W}^{(i)}}{\partial y_{j}}}{Y_{i}}=-C_{x},
\end{gathered}
$$

where $C_{x}$ denotes any constant with respect to $y$. Now, let us analyze each equality.

(i) Equality (B.25) with $(i, j)=(1,2)$. We divide the previous equality by $\mathfrak{l}_{11}^{(3)}$ (which does not depend on $\left.y_{2}\right)$ and get

$$
{\overline{\frac{\partial \mathcal{W}^{(1)}}{\partial y_{1}}}}^{Y_{2}}=1-C_{x}^{(1)}\left(\mathscr{\ell}_{11}^{(3)}\right)^{-1}
$$

Integrating with respect to $y_{1}$, we get

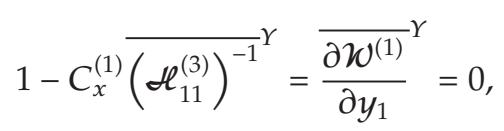

due to the periodicity of $\mathcal{W}^{(1)}$ in the $y_{1}$ variable. Thus,

$$
C_{x}^{(1)}=\left(\overline{\left(\mathscr{\ell}_{11}^{(3)}\right)^{-1}}\right)^{-1}=\left(\overline{\left({\overline{h_{[12]}^{3}}}^{Z}\right)^{-1}}\right)^{-1} .
$$


(ii) Equality (B.25) with $(i, j)=(2,1)$. We average the equality with respect to $y_{2}$ so that

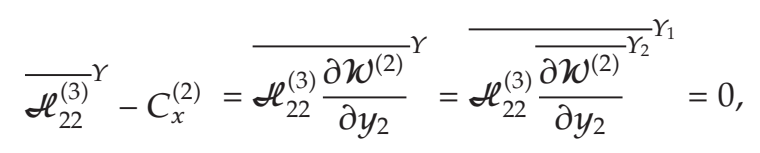

due to the periodicity of $\mathfrak{W}^{(2)}$ in the $y_{2}$ variable. We thus obtain

$$
C_{x}^{(2)}={\overline{\mathscr{H}_{22}^{(3)}}}^{Y}={\overline{\left({\overline{h_{[12]}^{-3}}}^{-Z}\right)^{-1}}}^{Y}
$$

(iii) Equality (B.26) with $(i, j)=(1,2)$. Integrating the equality with respect to $y_{2}$, we get

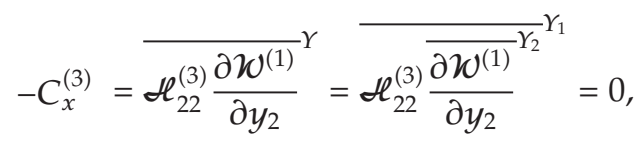

due to the periodicity of $\mathcal{W}^{(1)}$ in the $y_{2}$ variable. We conclude that $C_{x}^{(3)}=0$.

(iv) Equality (B.26) with $(i, j)=(2,1)$. Dividing the equality by $\mathscr{H}_{11}^{(3)}$ (which does not depend on the variable $y_{2}$ ) and integrating with respect to $y_{1}$, we get

$$
-C_{x}^{(4)}{\overline{\left(\mathscr{\ell}_{11}^{(3)}\right)^{-1}}}^{Y}={\overline{\frac{\partial \mathcal{W}^{(2)}}{\partial y_{1}}}}^{Y}=0,
$$

due to the periodicity of $\mathcal{W}^{(2)}$ in the $y_{1}$ variable. We conclude that $C_{x}^{(4)}=0$.

The coefficients $C_{x}^{(i)}$ exactly define the matrix $\mathcal{A}$ as

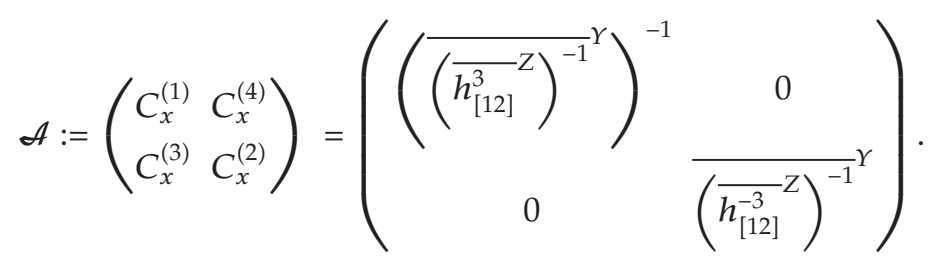

Step 5 (computation of the vector $\boldsymbol{B}^{0}={\overline{\mathscr{\ell}^{0}-\mathscr{\ell}^{(3)} \cdot \nabla_{y} \beta^{0}}}^{Y}$ ). Let us recall that, by definition, $\beta^{0}$ satisfies, for a.e. $x \in \Omega$,

$$
\int_{Y} \mathscr{L}^{(3)} \cdot \nabla_{y} \beta^{0} \nabla_{y} \psi=\int_{Y} \mathscr{L}^{0} \nabla_{y} \psi, \quad \forall \psi \in H_{\#}^{1}(Y) .
$$

Due to the simplifications based on the particular choice for $h$, we obtain

$$
\int_{Y} \mathscr{\ell}^{(3)} \cdot \nabla_{y} \beta^{0} \nabla_{y} \psi=\int_{Y} \mathscr{\ell}_{1}^{0} \frac{\partial \psi}{\partial y_{1}}, \quad \forall \psi \in H_{\#}^{1}(Y)
$$


If we use a test function only depending on $y_{1}$ or $y_{2}$, we get

$$
\begin{aligned}
& \int_{Y_{1}}{\overline{\mathscr{H}_{11}^{(3)}} \frac{\partial \beta^{0}}{\partial y_{1}}}_{Y_{2}}^{\frac{d \psi}{d y_{1}}}=\int_{Y_{1}} \overline{\mathscr{\ell}}_{1}^{0} Y_{2} \frac{d \psi}{d y_{2}}, \quad \forall \psi \in H_{\#}^{1}\left(Y_{1}\right),
\end{aligned}
$$

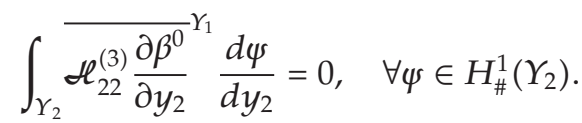

These two equations lead to the following equalities, which hold for a.e. $x \in \Omega$ :

$$
\begin{gathered}
{\overline{\mathscr{L}_{11}^{(3)} \frac{\partial \beta^{0}}{\partial y_{1}}}}^{\gamma_{2}}={\overline{\mathscr{H}_{1}^{0}}}^{\Upsilon_{2}}-D_{x,} \\
\frac{\mathscr{L}_{22}^{(3)} \frac{\partial \beta^{0}}{\partial y_{2}}}{Y_{1}}=-D_{x},
\end{gathered}
$$

where $D_{x}$ denotes any constant with respect to $y$. Now, let us analyze each equality.

(a) Equality (B.38). We divide the previous equality by $\mathscr{H}_{11}^{(3)}$ and get

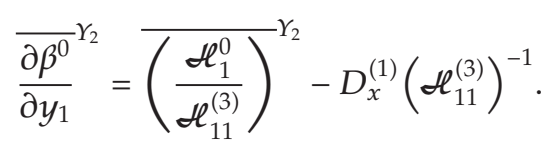

Integrating with respect to $y_{1}$, we get

$$
{\overline{\left(\frac{\mathscr{L}_{1}^{0}}{\mathscr{\ell}_{11}^{(3)}}\right.}}^{Y}-D_{x}^{(1)}{\overline{\left(\mathscr{\ell}_{11}^{(3)}\right)^{-1}}}^{Y}={\overline{\frac{\partial \beta^{0}}{\partial y_{1}}}}^{Y}=0,
$$

due to the periodicity of $\beta^{0}$ in the $y_{1}$ variable. Thus,

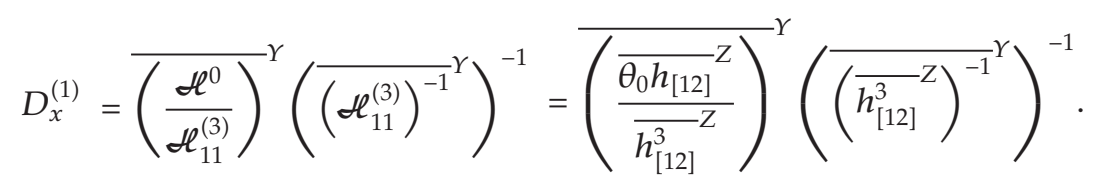

(b) Equality (B.39). Integrating the equality with respect to $y_{2}$, we get

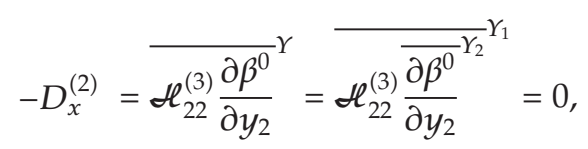

due to the periodicity of $\beta^{0}$ in the $y_{2}$ variable. We conclude that $D_{x}^{(2)}=0$. 
The coefficients $D_{x}^{(i)}$ exactly define the vector $B^{0}$ as

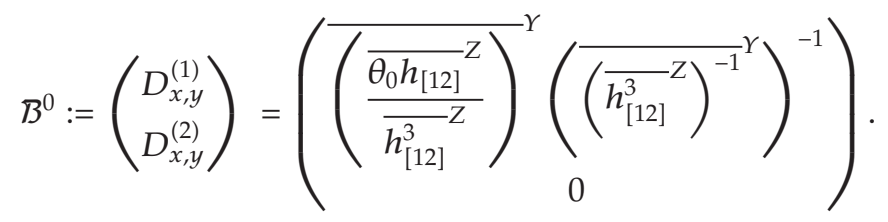

Step 6 (computation of the vector $\boldsymbol{B}^{\star}=\overline{\mathfrak{L}^{\star}-\mathscr{L}^{(3)} \cdot \nabla_{y} \beta^{\star}}$ ). The procedure follows the one explained in Step 5 (replacing $\mathscr{L}^{0}$ by $\mathscr{L}^{\star}$ and $\beta^{0}$ by $\beta^{\star}$ ). We obtain

$$
\left.B^{\star}=\left(\overline{\left(\frac{{\overline{h_{[12]}}}_{\overline{h_{[12]}^{3}}}^{Z}}{}\right)^{Y}} \underset{0}{\left({\overline{h_{[12]}^{3}}}^{Z}\right)^{-1}}\right)^{-1}\right) .
$$

As a consequence, the definition of the macroscopic saturation $\Theta=\mathbb{B}_{1}^{0} / \mathbb{B}_{1}^{\star}$ allows to recover the formula given in Table 1.

\section{Acknowledgments}

The author is very grateful to D.Cioranescu for fruitful discussions on periodic unfolding methods, and would like to thank also G. Bayada and C. Vázquez for many reasons.

\section{References}

[1] O. Reynolds, "On the theory of lubrication and its application to Mr. Beauchamp tower's experiments, including an experimental determination of the viscosity of olive oil," Philosophical Transactions of the Royal Society of London A, vol. 117, no. 1886, pp. 157-234, 1886.

[2] G. Capriz and G. Cimatti, "Partial lubrication of full cylindrical bearings," ASME Journal of Lubrication Technology, vol. 105, pp. 84-89, 1983.

[3] J. Coyne and H. G. Elrod, "Conditions for the rupture of a lubricating film: part 1," ASME Journal of Lubrication Technology, vol. 92, pp. 451-456, 1970.

[4] J. Coyne and H. G. Elrod, "Conditions for the rupture of a lubricating film: part 2," ASME Journal of Lubrication Technology, vol. 93, pp. 156-167, 1971.

[5] H. G. Elrod and M. L. Adams, "A computer program for cavitation," in Cavitation and Related Phenomena in Lubrication, pp. 37-42, Mechanical Engineering Publications, New York, NY, USA, 1975.

[6] N. Patir and H. S. Cheng, "An average flow model for determining effects of three-dimensional roughness on partial hydrodynamic lubrication," ASME Journal of Lubrication Technology, vol. 100, pp. 12-17, 1978.

[7] M. Jai, "Homogenization and two-scale convergence of the compressible Reynolds lubrication equation modelling the flying characteristics of a rough magnetic head over a rough rigid-disk surface," RAIRO Modélisation Mathématique et Analyse Numérique, vol. 29, no. 2, pp. 199-233, 1995.

[8] G. Bayada and J.-B. Faure, "A double-scale analysis approach of the Reynolds roughness. Comments and application to the journal bearing," ASME Journal of Tribology, vol. 111, pp. 323-330, 1989.

[9] G. Bayada and M. Chambat, "The transition between the Stokes equations and the Reynolds equation: a mathematical proof," Applied Mathematics and Optimization, vol. 14, no. 1, pp. 73-93, 1986.

[10] S. J. Alvarez and J. Carrillo, "A free boundary problem in theory of lubrication," Communications in Partial Differential Equations, vol. 19, no. 11-12, pp. 1743-1761, 1994.

[11] S. Martin, "Contribution à la modélisation de phénomènes de frontière libre en mécanique des films minces," Thèse de doctorat de l’Institut National des Sciences Appliquées de Lyon, Lyon, France, 2005. 
[12] C. Vázquez Cendón, "Existence and uniqueness of solution for a lubrication problem with cavitation in a journal bearing with axial supply," Advances in Mathematical Sciences and Applications, vol. 4, no. 2, pp. 313-331, 1994.

[13] H. W. Alt, "Numerical solution of steady-state porous flow free boundary problems," Numerische Mathematik, vol. 36, no. 1, pp. 73-98, 1980.

[14] G. Bayada, M. Chambat, and C. Vázquez, "Characteristics method for the formulation and computation of a free boundary cavitation problem," Journal of Computational and Applied Mathematics, vol. 98, no. 2, pp. 191-212, 1998.

[15] L. D. Marini and P. Pietra, "Fixed-point algorithms for stationary flow in porous media," Computer Methods in Applied Mechanics and Engineering, vol. 56, no. 1, pp. 17-45, 1986.

[16] H. W. Alt, "Strömungen durch inhomogene poröse Medien mit freiem Rand," Journal für die Reine und Angewandte Mathematik, vol. 305, pp. 89-115, 1979.

[17] J.-F. Rodrigues, "Some remarks on the homogenization of the dam problem," Manuscripta Mathematica, vol. 46, no. 1-3, pp. 65-82, 1984.

[18] A. Bermúdez and J. Durany, "La méthode des caractéristiques pour les problèmes de convectiondiffusion stationnaires," RAIRO Modélisation Mathématique et Analyse Numérique, vol. 21, no. 1, pp. 7-26, 1987.

[19] D. Cioranescu, A. Damlamian, and G. Griso, "Periodic unfolding and homogenization," Comptes Rendus de l'Académie des Sciences-Mathématique, vol. 335, no. 1, pp. 99-104, 2002.

[20] T. Arbogast, J. Douglas Jr., and U. Hornung, "Derivation of the double porosity model of single phase flow via homogenization theory," SIAM Journal on Mathematical Analysis, vol. 21, no. 4, pp. 823-836, 1990.

[21] G. Nguetseng, "A general convergence result for a functional related to the theory of homogenization," SIAM Journal on Mathematical Analysis, vol. 20, no. 3, pp. 608-623, 1989.

[22] G. Allaire, "Homogenization and two-scale convergence," SIAM Journal on Mathematical Analysis, vol. 23, no. 6, pp. 1482-1518, 1992.

[23] G. Allaire and M. Briane, "Multiscale convergence and reiterated homogenisation," Proceedings of the Royal Society of Edinburgh A, vol. 126, no. 2, pp. 297-342, 1996.

[24] D. Lukkassen, G. Nguetseng, and P. Wall, "Two-scale convergence," International Journal of Pure and Applied Mathematics, vol. 2, no. 1, pp. 35-86, 2002. 


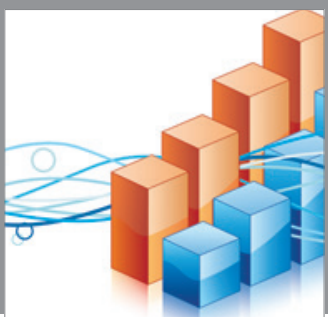

Advances in

Operations Research

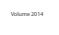

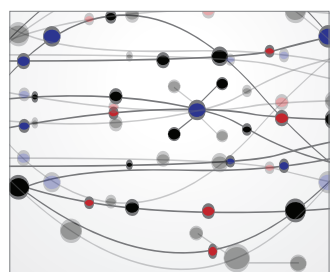

\section{The Scientific} World Journal
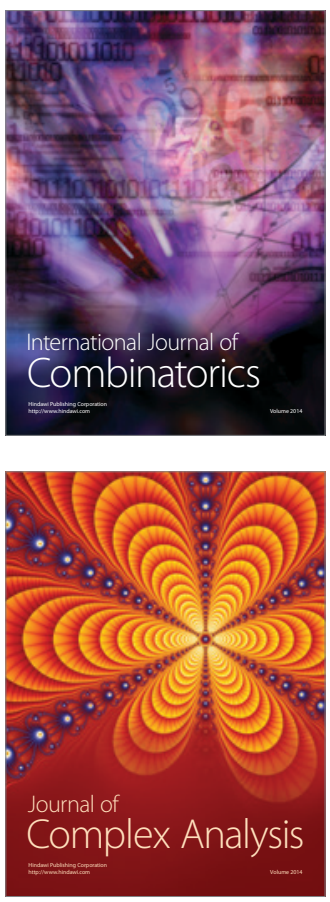

International Journal of

Mathematics and

Mathematical

Sciences
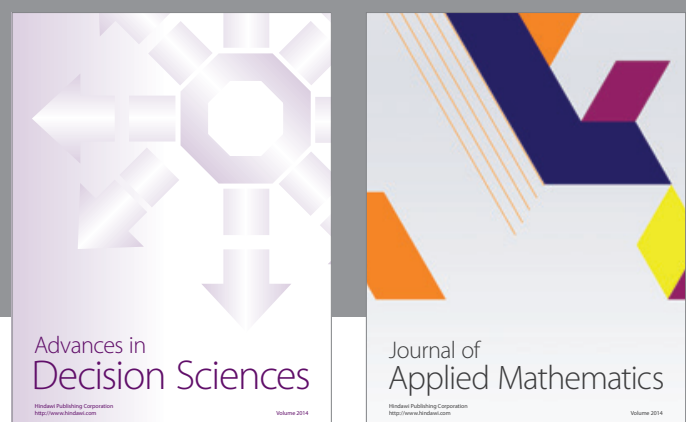

Journal of

Applied Mathematics
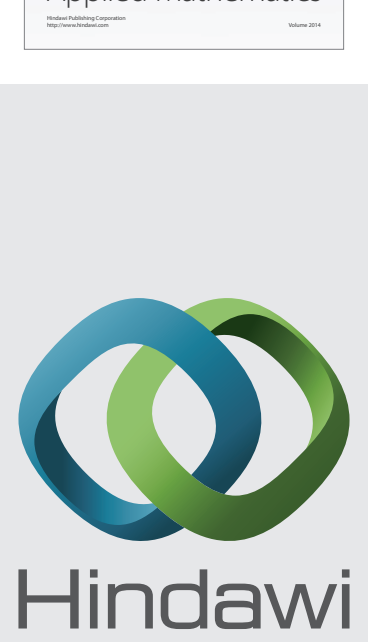

Submit your manuscripts at http://www.hindawi.com
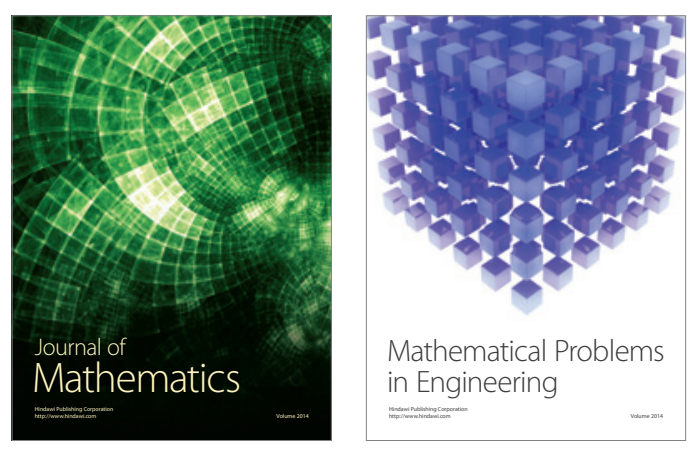

Mathematical Problems in Engineering
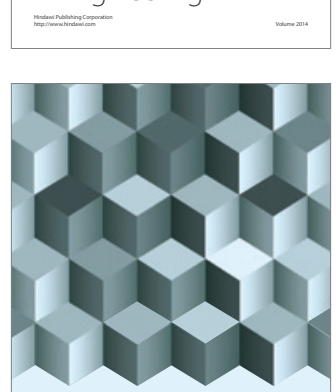

Journal of

Function Spaces
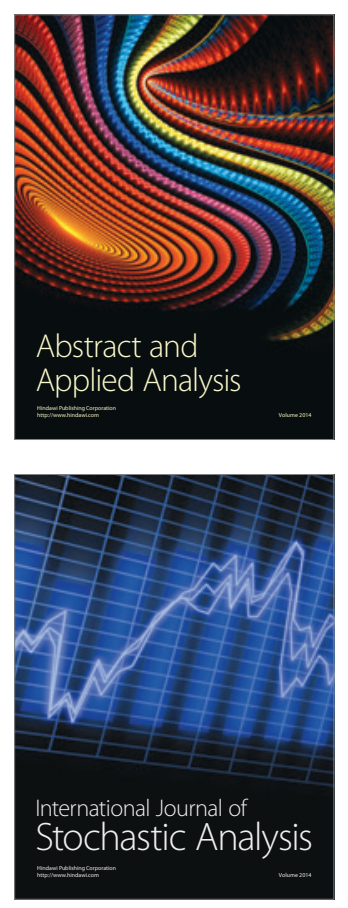

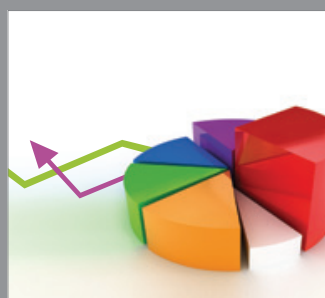

ournal of

Probability and Statistics

Promensencen
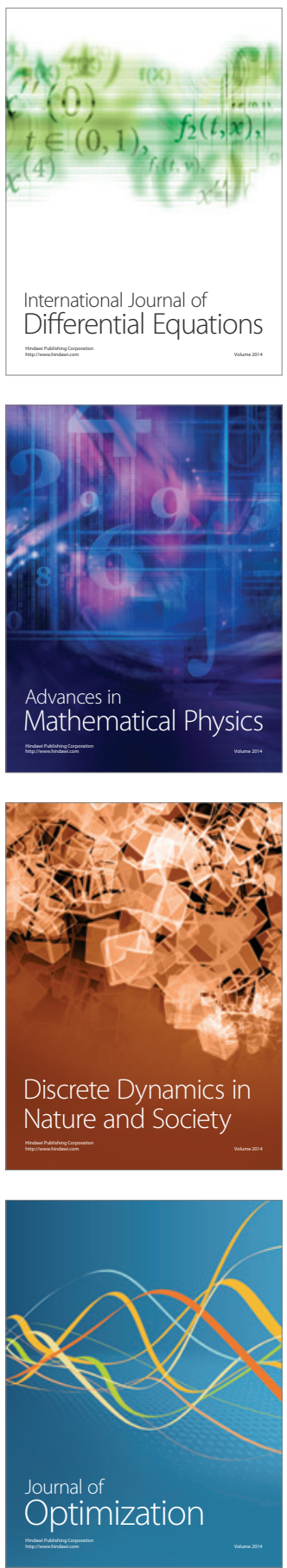